\title{
Benign Notochordal Cell Tumor of the Sacrum with Atypical Imaging Features: The Value of CT Guided Biopsy for Diagnosis
}

\author{
Dario Pasalic ${ }^{1, *}$, Patrick H. Luetmer ${ }^{2}$, Christopher H. Hunt ${ }^{2}$, Peter S. Rose ${ }^{3}$, Felix E. Diehn ${ }^{2}$, Andrew \\ L. Folpe ${ }^{4}$ and Doris E. Wenger ${ }^{2}$ \\ ${ }^{I}$ Mayo Medical School, Mayo Clinic, Rochester, MN 55905, USA \\ ${ }^{2}$ Department of Radiology, Mayo Clinic, Rochester, MN 55905, USA \\ ${ }^{3}$ Department of Orthopedic Surgery, Mayo Clinic, Rochester, MN 55905, USA \\ ${ }^{4}$ Department of Laboratory Medicine and Pathology, Mayo Clinic, Rochester, MN 55905, USA
}

\begin{abstract}
We report a case of a benign notochordal cell tumor (BNCT) of the sacrum with atypical imaging features, which was incidentally discovered in a 74-year-old man undergoing evaluation for progressively worsening hip and back pain. It is important for radiologists, pathologists and orthopedic surgeons to be aware of the diagnosis of BNCT and be familiar with its radiographic features to avoid unnecessary treatment. This case illustrates the advantage of percutaneous computed tomography (CT)-guided biopsy as a minimally invasive technique for definitive diagnosis of a BNCT with atypical imaging features.
\end{abstract}

Keywords: Benign notochordal cell tumor, Benign chordoma, Giant notochordal hamartoma, Giant notochordal rest and Needle biopsy.

\section{INTRODUCTION}

Benign notochordal cell tumors (BNCTs), also referred to as giant notochordal hamartomas of intraosseous origin [1], giant notochordal rests [2], or benign chordomas [3], usually present in the midline of the clivus or vertebral bodies and are believed to be notochordal remnants [4]. BNCTs are often discovered incidentally on imaging studies performed for patients presenting with non-specific symptoms such as acute-on-chronic back pain, numbness, stiffness, and reduced range of motion [3]. Although only 22 cases of BNCT have been reported in the radiographic literature as of 2011 [5], an autopsy study suggests a much higher prevalence, with 26 intraosseous BNCTs identified in 100 random autopsies [6]. While some evidence suggests that a minority of cases may progress to chordoma [7,8], $\mathrm{BNCTs}$ are generally regarded as benign lesions requiring only conservative management. In contrast, chordomas are malignant neoplasms treated with wide resection and/or particle beam radiation $[9,10]$. It is thus important for radiologists to be familiar with BNCTs and to recognize imaging features that can be utilized to differentiate them from chordomas.

Herein we report the clinicopathological and imaging features of an incidental BNCT of the sacrum that demonstrated atypical radiographic features and discuss the radiographic and pathological distinction of BNCT from chordoma. This case illustrates the value of a minimally invasive CT-guided needle biopsy in the workup of problematic notochordal lesions.

*Address correspondence to this author at the Mayo Clinic, 200 First Street SW, Rochester, MN, USA; Tel: (602)-384-7773;

E-mail: pasalic.dario@mayo.edu

\section{CASE REPORT}

A 74-year-old man with a long-standing history of hip and back pain presented for evaluation of progressively worsening symptoms. Nonsteroidal anti-inflammatory drugs, narcotics, and physical therapy resulted in pain reduction from a debilitating $9 / 10$ to a manageable $6 / 10$ on a visual analogue pain scale. However, the patient experienced difficulty going up stairs, gait imbalance, progressive weakness, and bowel and bladder incontinence, prompting him to pursue further medical intervention.

Unenhanced magnetic resonance imaging (MRI) of the lumbar spine with dedicated images of the sacrum (Fig. 1) showed a lesion in the S3 segment that extended to the superior aspect of S4. The lesion had a lobulated morphology and demonstrated intermediate signal on T1-weighted and heterogeneous signal on T2-weighted images, with areas of intermediate and high signal. In addition, the lesion contained tiny round foci of increased $\mathrm{T} 1$ signal that suppressed on T2-weighted images with fat suppression, indicative of a small component of intralesional fat. Although the lesion was predominantly intraosseous, there was focal permeation through the posterior cortex with a tiny nodular soft tissue mass in the adjacent spinal canal. Intravenous gadolinium was not administered due to patient claustrophobia, which precluded additional image acquisitions. At this time, the differential diagnosis included BNCT, chordoma, hemangioma, metastatic carcinoma, chondrosarcoma, myeloma, or lymphoma.

Unenhanced $\mathrm{CT}$ of the sacrum demonstrated diffuse subtle sclerosis in the lesion without evidence of a destructive osteolytic component (Fig. 2). This finding along with the foci of intralesional fat noted on MRI strongly 


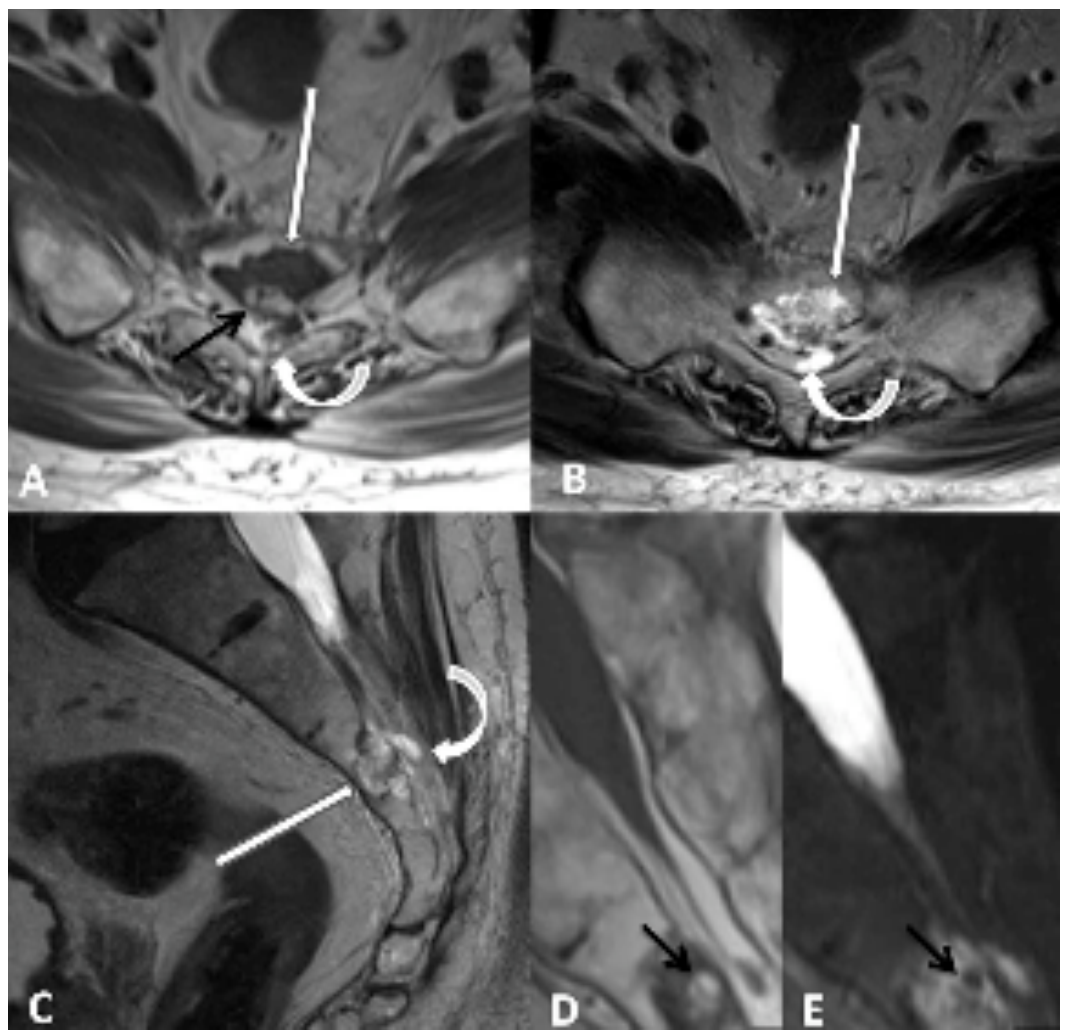

Fig. (1). Axial T1-weighted (A), axial T2- weighted (B), and sagittal T2-weighted (C) images demonstrate a lobulated mass involving S3 and the dorsal superior body of L4 (straight white arrows) with heterogeneous T1- and T2 signal with areas of intermediate and high signal. There is subtle focal dorsal extraosseous extension of the tumor into the sacral canal (curved arrows). Subtle marrow edema along the ventral and superior aspect of the tumor margin is noted on sagittal T2-weighted image (C). Magnified sagittal T1- (D) and fat suppressed sagittal T2-weighted (E) images as well as axial T1- weighted image (A) demonstrate lobulated foci of intralesional fat (black arrows) with complete suppression of fat signal on fat suppressed T2-weighted images (E).

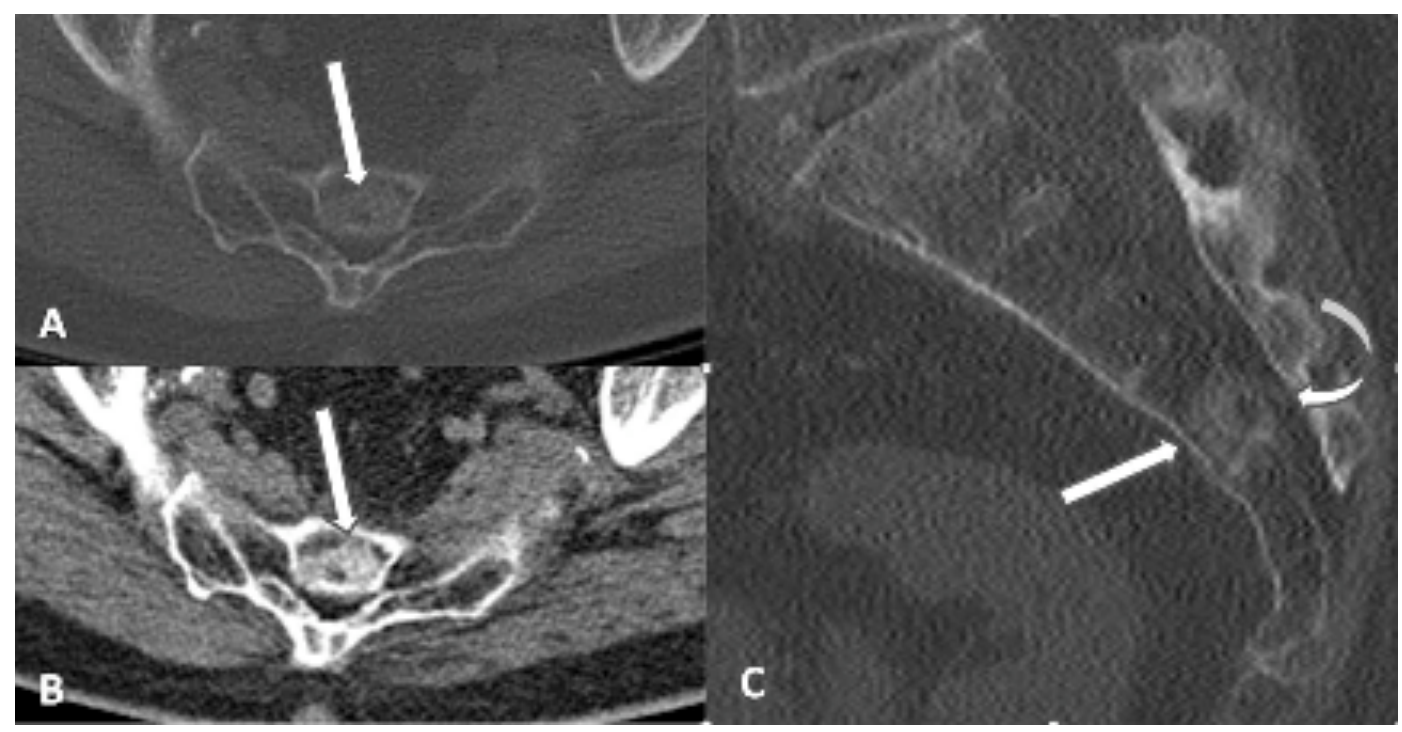

Fig. (2). CT images of the sacrum with axial bone (A) and soft tissue (B) algorithm and sagittal bone (C) algorithm demonstrate a sclerotic lesion within S3 and the superior body of S4 (white arrows), which is diffusely sclerotic with a relatively narrow zone of transition. However, there is subtle focal cortical permeation involving the cortex along the dorsal aspect of the lesion, with subtle extraosseous extension into the sacral canal (curved arrow).

favored a diagnosis of BNCT. However, given the focal permeation of tumor through the dorsal cortex forming a tiny nodular extraosseous soft tissue mass, the lesion was considered atypical for BNCT, and a biopsy was recommended for definitive histologic diagnosis.
Following interdisciplinary discussion, a decision was made to perform a minimally invasive percutaneous CTguided biopsy rather than pursue a metastatic work-up.

CT-guided biopsy (Fig. 3) was performed with the patient in an oblique prone position on the $\mathrm{CT}$ table with the 


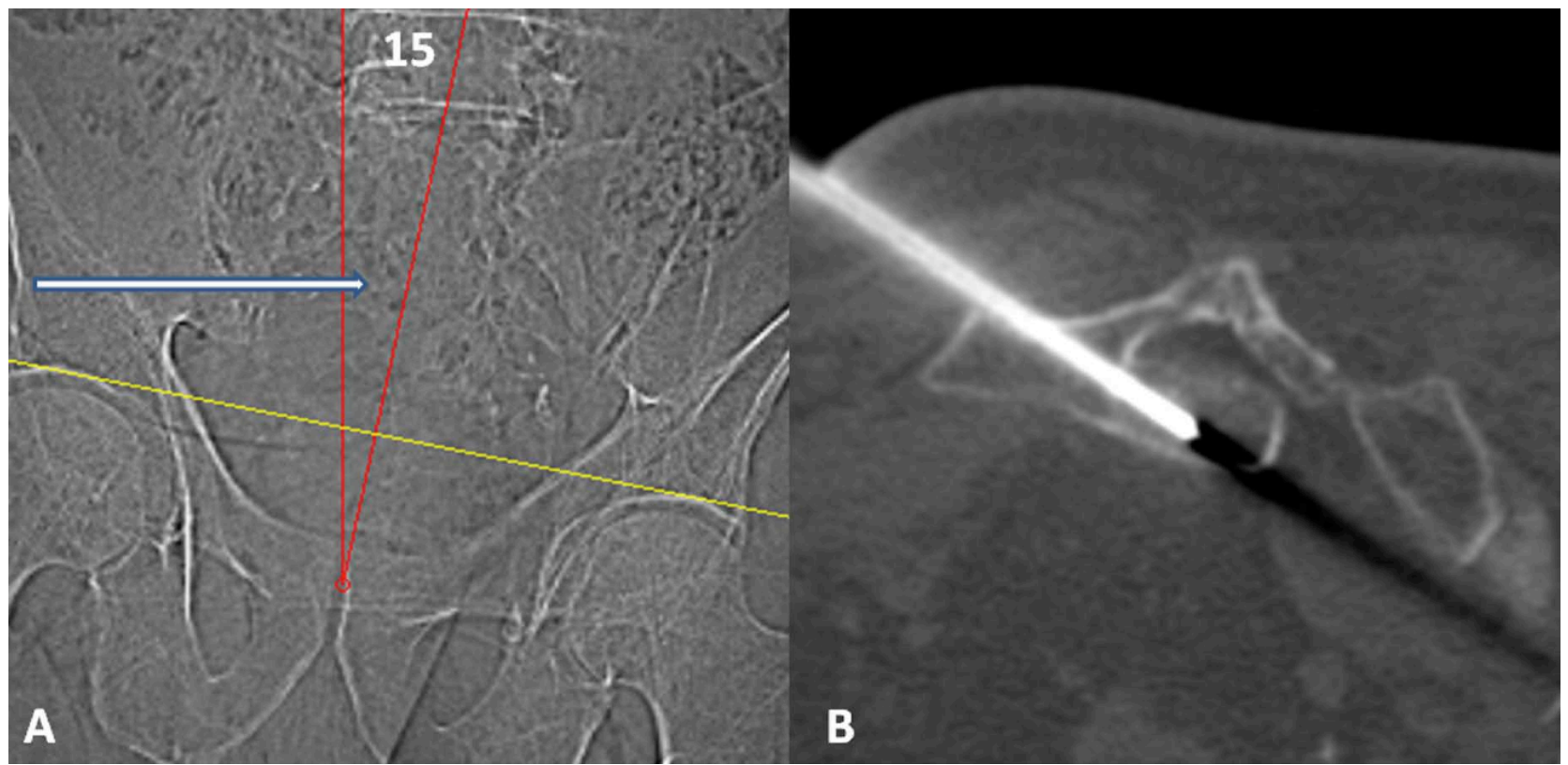

Fig. (3). Scout image (A) with patient prone demonstrates oblique positioning with spine and pelvis positioned approximately 15 degrees to the right (red angle). This aligned the left S4 pedicle with the scanner gantry (white arrow.) Procedural CT image (B) demonstrates an 11gauge needle extending through the left S4 pedicle into the intraosseous tumor at S3.

spine and pelvis positioned 15 degrees to the right to align the left S4 pedicle with the gantry. This position facilitated an uneventful biopsy of the intraosseous tumor component at S3 via a left S4 transpedicular approach using coaxial technique with 3 cores obtained using a trephinated 14 gauge needle and one final core obtained with an 11-gauge needle. Although lesions in which chordoma is the leading differential diagnosis are traditionally approached with a (near) midline biopsy tract, this tract was deliberately selected to allow best sampling of a tumor in which the role of the biopsy was to confirm the imaging impression of BNCT. Additionally, in the unlikely event that a chordoma was diagnosed, definitive treatment in this 74 year old gentleman would be proton beam therapy in which the biopsy tract could be sterilized.

The submitted needle biopsies showed (Fig. 4) typical morphological features of BNCT, with a hypocellular proliferation of bland, eosinophilic, epithelioid cells, often showing striking cytoplasmic vacuolization, and mimicking adipose tissue. Morphological features of chordoma, such as myxoid matrix, cords and chains of epithelioid cells, physaliphorous cells, mitotic activity and necrosis, were absent. By immunohistochemistry the neoplastic cells were strongly positive for cytokeratins using the OSCAR monoclonal antibody and for the notochord-specific transcription factor brachyury.

At initial clinical follow-up, the BNCT was thought to be incidental to the pain syndrome. The patient will be followed with periodic MRI of the sacrum to ensure stability of the lesion.

\section{DISCUSSION}

BNCTs are often discovered incidentally and may present a diagnostic challenge. Familiarity with the diagnosis of BNCTs and their imaging features is critical in distinguishing them from chordomas or other malignant lesions. BNCTs are benign lesions which are typically followed conservatively with follow-up imaging [3], while the majority of chordomas are treated with wide surgical resection [11]. Recent studies [4] evaluating chordomas and BNCTs have found certain key differentiating features. BNCTs typically present as small midline lesions that are occult on radiographs, with no evidence of cortical destruction or soft tissue mass [12]. They show little if any activity on bone scan $[4,12]$. They may be occult on CT, but often demonstrate subtle hazy sclerosis without evidence of a destructive osteolytic component [12]. On MRI, BNCTs typically present as a small intraosseous mass without associated soft tissue mass. Often, there is a slightly nodular morphology but with nonspecific imaging features including intermediate signal on T1-weighted images, heterogeneous predominantly hyperintense signal on $\mathrm{T} 2$-weighted images and no enhancement with gadolinium $[4,12]$. Chordomas, in contrast, typically present as midline aggressive osteolytic destructive lesions with associated cortical destruction, soft tissue mass and may contain internal matrix calcification [4, 12]. The majority of chordomas also demonstrate increased activity on Tc 99-m nuclear scintigrams [13] and typically enhance with gadolinium [14]. An additional imaging feature that can be instrumental in differentiating between BNCT and chordoma is the presence of tiny foci of intralesional fat. The latter feature has been described in BNCTs and excludes the diagnosis of chordoma which virtually never contain intralesional fat $[1,3]$.

It is important to recognize the complimentary nature of MRI and CT for the evaluation of notochordal lesions. MR imaging provides superior sensitivity for lesion detection. However, due to the nonspecific signal characteristics of the majority of the lesions, it is essential to correlate the MRI findings with radiographs and preferably CT to identify patterns of bone destruction or lack thereof. Although there are some characteristic and distinguishing imaging features for BNCT, correlation with histopathology is crucial for 

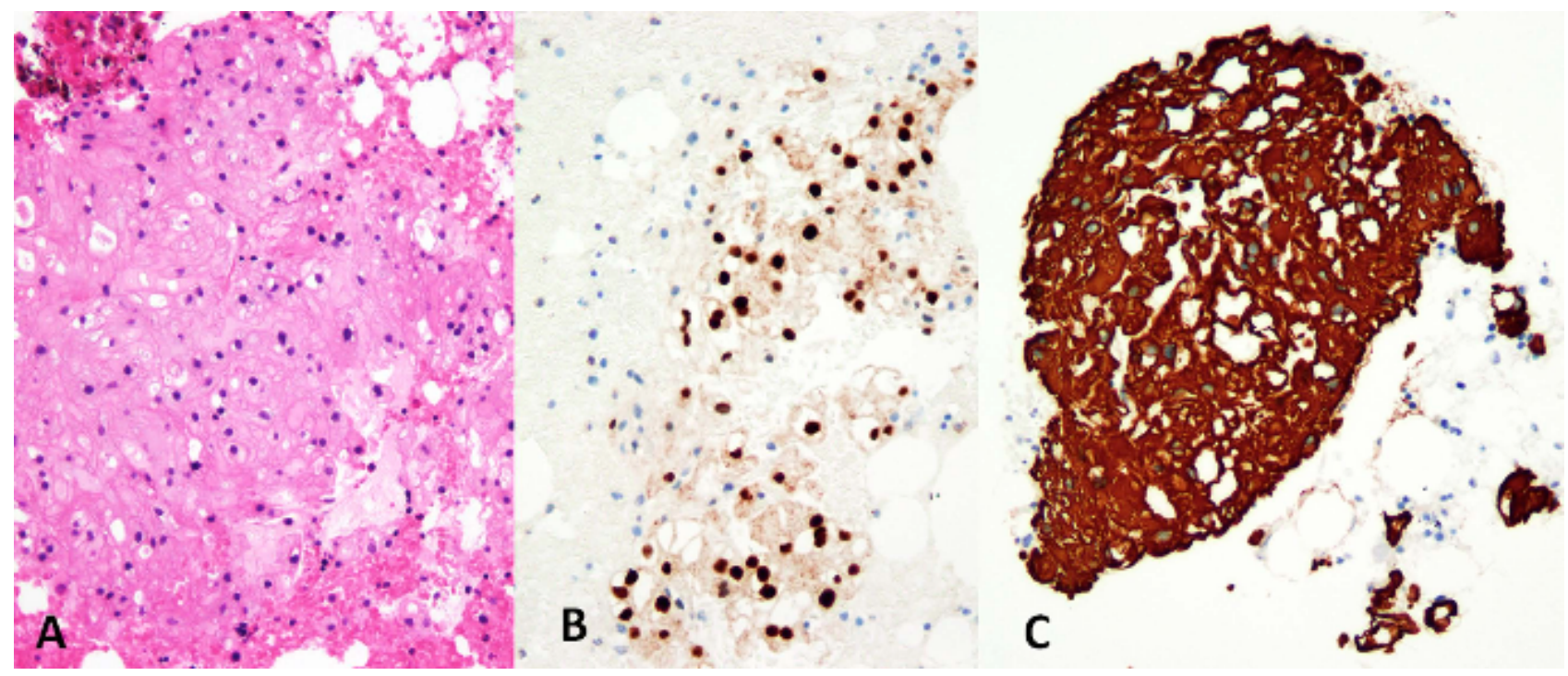

Fig. (4). BNCT, composed of bland, partially vacuolated, eosinophilic epithelioid cells (A) (Hematoxylin and eosin). The neoplastic cells expressed the notochordal marker brachyury (B) and cytokeratins $(\mathbf{C})$, confirming notochordal differentiation.

establishing an accurate diagnosis in atypical cases. Both histopathologic evaluation and immunohistochemical profiles play an important role in distinguishing between BNCTs and chordomas [15].

Since one of the distinctive imaging features between BNCT and chordoma is the lack of soft tissue mass with the former, the case of BNCT that we report is described as having atypical imaging features due to the presence of focal cortical permeation with an associated, albeit very small, epidural soft tissue mass. The authors know of an unpublished case of a BNCT with a soft tissue mass that was presented during the discussion of Case 47 at the International Skeletal Society (ISS) in Rome in 2012 by C.Y. Inwards and one of the authors (DEW). The latter case had identical imaging features to what we present, including location in the lower sacrum with a small associated soft tissue mass and pathological confirmation as a BNCT following complete surgical resection. In contradistinction, there is an additional case in the literature of a BNCT in the L1 vertebral body [16] with coexisting histologically proven chordoma forming a large epidural mass spanning two vertebral levels and causing compression of the conus medullaris.

Although not excluded, the possibility of a coexisting BNCT and chordoma is unlikely in our case given the pathologic confirmation of findings compatible with BNCT and the fact that there was only subtle cortical permeation with a very small associated soft tissue mass along with the presence of intralesional fat on MRI. The remaining clinical and imaging features in our case are similar to previously reported BNCTs, including initial incidental detection with MRI, negative radiographs and subtle sclerosis on CT without evidence of a destructive osteolytic component. However, given the proposed theory by some authors that BNCT may represent a precursor lesion for chordoma, substantiated by the previously described case report of a lesion with coexistent BNCT and chordoma [16], this lesion will be followed closely with periodic MRI to ensure stability.
While there are only limited case reports and small case series, BNCTs are likely more common than originally thought, given the high incidence of BNCT in an autopsy series [6]. In our experience, BNCTs are being encountered with increasing frequency with MRI and less often CT due to the increase in utilization of these imaging modalities for the evaluation of patients with back pain. It is important for radiologists, pathologists and orthopedic surgeons to be aware of the diagnosis of BNCT and to be familiar with its radiographic features to avoid unnecessary treatment. This case illustrates the role of a percutaneous CT-guided biopsy as a minimally invasive technique for definitive diagnosis of a BNCT with atypical imaging features.

\section{CONFLICT OF INTEREST}

The authors confirm that this article content has no conflicts of interest.

\section{ACKNOWLEDGEMENTS}

Declared none.

\section{REFERENCES}

[1] Mirra JM, Brien EW. Giant notochordal hamartoma of intraosseous origin: a newly reported benign entity to be distinguished from chordoma: Report of two cases. Skeletal Radiol 2001; 30(12): 698709 .

[2] Darby AJ, Cassar-Pullicino VN, McCall IW, et al. Vertebral intraosseous chordoma or giant notochordal rest? Skeletal Radiol 1999; 28(6): 342-6.

[3] Amer HZ, Hameed M. Intraosseous benign notochordal cell tumor. Arch Pathol Lab Med 2010; 134(2): 283-8.

[4] Nishiguchi T, Mochizuki K, Ohsawa M, et al. Differentiating benign notochordal cell tumors from chordomas: radiographic features on MRI, CT, and tomography. AJR Am J Roentgenol 2011; 196(3): 644-50.

[5] Kyriakos M. Benign notochordal lesions of the axial skeleton: a review and current appraisal. Skeletal Radiol 2011; 40(9): 1141-52.

[6] Yamaguchi T, Suzuki S, Ishiiwa H, Ueda Y. Intraosseous benign notochordal cell tumours: overlooked precursors of classic chordomas? Histopathology 2004; 44(6): 597-602.

[7] Yamaguchi $\mathrm{T}$, Watanabe-Ishiiwa $\mathrm{H}$, Suzuki $\mathrm{S}$, et al. Incipient chordoma: a report of two cases of early-stage chordoma arising 
from benign notochordal cell tumors. Mod Pathol 2005; 18(7): 1005-10.

[8] Shen J, Li CD, Yang HL, et al. Classic chordoma coexisting with benign notochordal cell rest demonstrating different immunohistological expression patterns of brachyury and galectin3. J Clin Neurosci 2011; 18(1): 96-9.

[9] Chen YL, Liebsch N, Kobayashi W, et al. Definitive high-dose photon/proton radiotherapy for unresected mobile spine and sacral chordomas. Spine (Phila Pa 1976) 2013; 38(15): E930-6.

[10] Fuchs B, Dickey ID, Yaszemski MJ, Inwards CY, Sim FH. Operative management of sacral chordoma. J Bone Joint Surg Am 2005; 87(10): 2211-6.

[11] Walcott BP, Nahed BV, Mohyeldin A, et al. Chordoma: current concepts, management, and future directions. Lancet Oncol 2012; 13(2): e69-76.
[12] Yamaguchi T, Iwata J, Sugihara S, et al. Distinguishing benign notochordal cell tumors from vertebral chordoma. Skeletal Radiol 2008; 37(4): 291-9.

[13] Papagelopoulos PJ, Mavrogenis AF, Galanis EC, et al. Chordoma of the spine: clinicopathological features, diagnosis, and treatment. Orthopedics 2004; 27(12): 1256-63; quiz 1264-5.

[14] Sung MS, Lee GK, Kang HS, et al. Sacrococcygeal chordoma: MR imaging in 30 patients. Skeletal Radiol 2005; 34(2): 87-94.

[15] Yamaguchi T, Yamato M, Saotome K. First histologically confirmed case of a classic chordoma arising in a precursor benign notochordal lesion: differential diagnosis of benign and malignant notochordal lesions. Skeletal Radiol 2002; 31(7): 413-8.

[16] Nishiguchi T, Mochizuki K, Tsujio T, et al. Lumbar vertebral chordoma arising from an intraosseous benign notochordal cell tumour: radiological findings and histopathological description with a good clinical outcome. Br J Radiol 2010; 83(987): e49-5.

(C) Pasalic et al.; Licensee Bentham Open.

This is an open access article licensed under the terms of the Creative Commons Attribution Non-Commercial License (http://creativecommons.org/licenses/by$\mathrm{nc} / 3.0 /$ ), which permits unrestricted, non-commercial use, distribution and reproduction in any medium, provided the work is properly cited. 